\title{
Discurso do Sujeito Coletivo aplicado a medição da motivação dos alunos durante a execução de atividades
}

Sam da S. Devincenzi, UNIPAMPA, sam.devincenzi@gmail.com

Viviani Rios Kwecko, IFRS, viviani.kwecko@riogrande.ifrs.edu.br

Fernando Pereira de Tolêdo, FURG, ftoledo@furg.br

Gabriel Moraes, FURG, g.moraes@furg.br

Silvia Silva da Costa Botelho, FURG, silviacb@furg.br

Resumo: Neste estudo buscamos compreender como a motivação dos estudantes a aprendizagem pode ser afetada por envio de gatilhos motivacionais automáticos durante a execucão das atividades em ambientes de Aprendizagem Baseada em Problemas (PBL). A metodologia para validação dos gatilhos foi desenvolvida em dois momentos: (i) em 2017 com a participação de 60 alunos do curso de Engenharia de Software; e (ii) em $2018 \mathrm{com}$ 23 alunos do segundo ano do curso de Engenharia de Computação. Ao final de cada um desses estudos, os pontos de vista dos alunos foram agrupados por classe e expressos através da construção do Discurso do Sujeito Coletivo (DSC). Assim, este estudo contribui para a discussão e apresentação do DSC como abordagem capaz de auxiliar os professores durante a avaliação das atividades propostas em sala de aula, principalmente por possibilitar observar a opinião de um grupo como um único sujeito.

Palavras-chave: PBL, DSC, motivação, aluno, Aprendizagem Baseada em Problemas

\section{Collective Subject Discourse applied to measure student motivation in activities execution}

Abstract. In this study we seek to understand how students' motivation to learn can be affected by sending automatic motivational triggers (sounds, notifications, etc.) during the execution of activities in environments of Problem Based Learning (PBL). The methodology for the validation of the triggers was developed in two moments: (i) in 2017 with the participation of 60 students of the Software Engineering course; and (ii) in 2018 with 23 students of the second year of the Computer Engineering course. At the end of each of these studies, students' views were grouped by class and expressed through the construction of the Collective Subject Discourse (DSC). Thus, this study contributes to the discussion and presentation of the DSC as an approach capable of assisting teachers during the evaluation of the activities proposed in the classroom, mainly because it makes it possible to observe the opinion of a group as a single subject.

Keywords: PBL, DSC, motivation, student, Problem Based Learning

\section{Introdução}

Aprendizagem Baseada em Problemas (Problem-Based Learning - PBL) é uma das estratégias de Aprendizagem Ativa que busca incentivar a construção do conhecimento de estudantes através do uso de problemas do mundo real para motivá-lo (Barrows, 1986). A PBL valoriza a importância da prática, focando a aprendizagem experiencial em torno da investigação, explicação e resolução de problemas significativos (Hmelo-Silver, 2004). Porém paraque o processo PBL tenha sucesso, a principal premissa é a qualidade do 
problema a ser utilizado no processo de motivação à aprendizagem dos alunos. Segundo (Hmelo-Silver, 2004) um bom problema deve ser complexo, está em aberto e ser realista com relação a experiência do aluno. A complexidade envolvida na solução do problema deve ser suficiente para exigir que conteúdos sejam inter-relacionados, motivando assim a necessidade do aluno em conhecer diferentes assuntos. Esta multidisciplinaridade ajuda a construir um conhecimento extensivo e reflexivo. Além da motivação do aluno para resolução do problema, a PBL depende da intervenção do professor que deve atuar como facilitador, orientando a aprendizagem do aluno através das etapas de fatos, ideias, pontos de aprendizagem e plano de ação, descritas por (Hmelo-Silver, 2004). Cabe ao professor apresentar o problema para os alunos, com informações mínimas sobre o mesmo. Os alunos, por sua vez, devem investigar, entender e definir os fatos que compõem 0 problema, a fim de definir o que deve ser produzido, respondido e resolvido. À medida que os alunos compreendem melhor o problema a partir dos dados coletados, iniciam a identificar fontes, recursos, listar ações, gerar hipóteses registrando suas ideias para a solução do problema.

Uma etapa importante deste processo é a reflexão quanto aos conceitos necessários para resolver o problema. Os alunos devem conseguir identificar as deficiências de seus conhecimento, mapeando os pontos de aprendizagem para definir seu plano de ação. Nesta fase, o professor precisa estimular a auto-aprendizagem fornecendo um suporte justin-time ao aluno, que por sua vez, necessita encontrar o momento em que suas reflexões geram novos conhecimentos entre os membros dos grupos. Enquanto trabalham nesse processo, os alunos podem usar a metodologia do quadro branco (suporte às atividades do processo PBL) para anotar, compartilhar e organizar as informações que estão sendo geradas. Neste quadro, são registrados: (i) fatos: informações levantadas pelos alunos na declaração do problema; (ii) ideias: hipóteses trabalhadas durante o processo de resolução; (iii) problemas de aprendizagem: dúvidas sobre conceitos que necessitam de futuros estudos; (iv) plano de ação: planos para resolver o problema ou obter informações.

Sendo assim, nota-se que a PBL, é inteiramente dependente das atividades de reflexão e cooperação por parte dos alunos, visto que a capacidade de reflexão é fundamental para a construção de um conhecimento capaz de ser recuperado de um ambiente para a aplicação em outro. Conseguir que cooperação, reflexão e motivação sejam promovidas, cada vez mais na forma just-in-time (com intervenção adequada), é outro desafios da PBL (Hmelo-Silver, 2004). Quando não levadas em conta as peculiaridades do aluno em relação a suas preferências individuais sobre temas de trabalho, sua disponibilidade de tempo para se dedicar aos estudos e a infraestrutura de aprendizado disponível aumenta a possibilidade de desmotivação.

Diante desse contexto o uso de tecnologias móveis e ubíquas para suporte ao processo PBL (u-PBL) pode representar uma estratégia motivacional, tendo em vista que estas tecnologias possibilitam o aprendizado em qualquer lugar e hora no tempo do aluno (Santaella, 2014). A chamada u-PBL é uma abordagem da PBL que se baseia no uso combinado de ambientes para instruções dos professores, ferramentas para construção de atividades de aprendizado e ambiente de aprendizagem colaborativo ubíquo para estudantes, buscando assim, assegurar a aprendizagem significativa (Ku e Chang, 2010). Porém, (Devincenzi et al., 2018) destaca pontos de carências associados aos trabalhos da uPBL em relação a PBL, como: (a) falta de artefatos para incentivo a reflexão dos alunos, a 
cooperação entre eles e a motivação dos mesmos (Hwang et al., 2009); (b) fraco envolvimento das vivências do cotidiano do aluno (tempo e espaço) em seu processo educacional (Ku e Chang, 2010); e (c) falta de inferência inteligente do sistema, a partir do uso de tecnologias ubíquas para detecção do ambiente do aluno (Hung et al., 2011).

Outro ponto de interesse são os estudos sobre Tecnologia Persuasiva (TP) que segundo Fogg (2009) são sistemas computacionais utilizados para tentar alterar o comportamento humano. Para o autor esta mudança de comportamento ocorre através de um modelo denominado Fogg Behavior Model (FBM), o qual descreve a mudança de comportamento como sendo um produto de três fatores: i) motivação: facilitador para mudança de comportamento; ii) habilidade: grau de competência que um indivíduo tem para realizar um comportamento; e iii) gatilho: recurso responsável por incitar o processo de mudança de comportamento do indivíduo. Segundo Fog (2009), para um indivíduo alcançar um comportamento alvo, o mesmo deve ser notificado por meio de um gatilho (envio de mensagem, aviso, etc), estar motivado e ter habilidade de executar o comportamento. O comportamento alvo somente será alcançado na presença dos fatores motivação, habilidade e notificação ao indivíduo (gatilho) no instante de tempo adequado.

As pesquisas realizadas por (Devincenzi et al., 2017; 2018), destacam a utilização do FBM como estratégias para motivar a aquisição de conhecimentos e/ou habilidades em ambientes de aprendizagem. Já os trabalhos de (Filippou, 2015) e (Dijkstra, 2016) relacionam TPs ao aprimoramento das habilidades sociais, eficácia do aprendizado e sucesso acadêmico. Reconhecendo a capacidade das Tecnologias Ubíquas e Persuasivas em atuar sobre o comportamento dos indivíduos, desenvolvemos em 2018 um modelo de motivação individualizada (Devincenzi et al., 2018) baseado na integração do FBM (Fogg, 2009) ao processo PBL. O detalhamento deste modelo e de sua viabilidade tecnológica é apresentada em (Devincenzi et al., 2017) a partir das seguintes relações:

1. Entre as Etapas: Gatilho Facilitador, regra a evolução do processo, delimitado pelo professor, pelo tempo e/ou pela evolução do grupo;

2. Etapa 1: Desafio do Problema - Gatilho Faísca do tipo prazer e dor, condiciona o aluno à executar um comportamento no momento em que o desafio é recebido.

3. Etapa 2: Aprofundamento do Problema - Gatilho Faísca, incentiva a descoberta de conteúdos; Gatilho Facilitador, interpõe o aluno aos pontos de conhecimento envolvidos com o problema, revelando quais habilidades devem ser desenvolvidas;

4. Etapa 3: Gatilho Facilitador, possibilita ao aluno o desenvolvimento de habilidades no momento em que busca informações sobre o problema;

5. Etapa 4: Gatilho Facilitador, dispara para o aluno um processo de autoavaliação do conhecimento adquirido; Gatilho Faísca, disparado ao aluno no momento em que ele identifica quais os temas a aprender;

6. Etapa 5: Gatilho Facilitador, dispara ao aluno a necessidade de busca por conhecimentos necessários para a solução do problema;

7. Etapa 6: Gatilho Facilitador, instiga o aluno a colocar em prática os novos conhecimentos adquiridos; Gatilho Faísca, disparado ao aluno no momento que ele identifica a possibilidade de aplicação do novo "saber" para a solução do problema;

8. Etapa 7: Gatilho Faísca, disparado no momento em que o aluno apresenta a solução e identifica seu sucesso na atividade e sua capacidade de aplicar o conhecimento para a solução de problemas. 
Analisando o mapeamento exposto, podemos identificar que várias destas atividades (gatilhos) podem ser desempenhadas via computador, porém o simples uso do computador deixa lacunas no processo. Os disparos em momentos pré-definidos não levam em conta o contexto (Tempo, Local, Competência, Motivação, etc.), não interagindo com o aluno no momento adequado. Assim os benefícios do uso do FBM apontado por (Fogg, 2009) nestes momentos de disparo para se conseguir alcançar o comportamento desejado são minimizados. Assim, buscando uma forma de otimizar o comportamento persuasivo da tecnologia ubíqua, desenvolvemos um artefato tecnológico (denominado TutorApp), brevemente apresentado na próxima seção, que reúne o contexto do FBM às TP definidos em (Devincenzi et al., 2018) e a metodologia de quadro branco proposta por (Hmelo-Silver, 2004). Sendo que neste artigo limitamos a apresentação dos resultados alcançados na etapa de validação da aplicação proposta.

\section{Caminhos metodológicos}

Este é um estudo descritivo desenvolvido com o intuito de (i) compreender como a motivação pode ser afetada por gatilhos motivacionais automaticamente enviados pela aplicação (TutorApp) aos alunos durante a execução de atividades em sala de aula; e (ii) verificando se essas mensagens motivacionais e a estrutura da aplicação de alguma forma contribuem no processo de ensino-aprendizagem de uma metodologia PBL. Apresentamos um breve detalhamento da aplicação desenvolvida seguida do estudo de caso realizado.

\subsection{O aplicativo TutorApp}

O TutorApp, conforme mencionado, foi fundamentado nas observações realizadas por (Devincenzi et al., 2018), (Hmelo-Silver, 2004). A aplicação tem o intuito de intervir no nível de motivação dos alunos de modo a catalisar o seu processo de aprendizagem e apoiar a execução das práticas pedagógicas, sendo composta por dois ambientes :

1. Ambiente aluno: WebApp no qual o estudante: (a) tem acesso aos desafios organizados pelo professor; (b) recebe notificações (gatilhos) contendo os objetos de aprendizagem, (artigos, vídeos explicativos, reportagens, dicas, fontes de informação); e (c) registra e compartilha, em uma estrutura de quadro branco, suas dúvidas com os demais membros de sua turma ou grupo de trabalho.

2. Ambiente do professor: no qual o docente pode administrar e interagir com os alunos, efetuar o planejamento das disciplinas, bem como inserir planos de ensino, problemas e desafios e conteúdos a serem listados para os alunos. O professor em determinados momentos têm autonomia para intervir no processo de aprendizagem por meio de uma intervenção individual, por grupo ou turma.

A estrutura de gatilhos foi discretizada para aplicação em diferentes condições de uso da TP, considerando a percepção do aluno, o momento oportuno para o envio dos gatilhos, os níveis de habilidade e motivação. (Devincenzi et al., 2017). Foram implementados os seguintes gatilhos:

1. Gatilho Mediação Externa: disparo de mensagens programadas pelo professor

2. Gatilho Affordance: disparo baseado na percepção contextual realizada pelo sensoriamento do artefato tecnológico.

3. Gatilho Auto-aprendizagem: usado para disponibilizar aprendizagem próximas a 
atividade executada, de forma a evitar grandes desvios de pesquisa, que possam trazer elevado grau de desmotivação.

\subsection{O estudo de caso}

O estudo foi implementado nos cursos de graduação em computação de duas universidades brasileiras, por estes cursos utilizarem nas disciplinas acompanhadas a metodologia PBL. O aplicativo TutorApp interagiu com os alunos nos seguintes cenários:

1. Quando o aluno pesquisou via navegador ou registrou na estrutura "Quadro Branco" necessidades de auto-aprendizado, a aplicação enviou links para artigos de bases de conhecimento reconhecidas, garantindo assim a qualidade da fonte de informação;

2. Baseado na localização do aluno, identificada pelo seu ponto de GPS capturado de seu dispositivo móvel, foi disparada a indicação de um livro que aborda um tema que o aluno necessita aprender, no momento em que o mesmo está circulando próximo a área da biblioteca de sua universidade;

3. Quando Fatos do Problema são cadastrados no "Quadro Branco", a aplicação informou ao aluno data e hora de disciplinas onde os temas relacionados a estes Fatos serão vistos;

Estudo de Caso 1: Realizado no segundo semestre de 2017, contou com a participação de 60 alunos dos segundo e sexto semestres do curso de Engenharia de Software.

Estudo de Caso 2: Realizado no primeiro semestre de 2018, contou com a participação de 23 alunos do segundo ano do curso de Engenharia da Computação.

Para a análise dos experimentos, após cada estudo ser concluído, a opinião dos alunos, agrupada por turma, foi expressa com a construção do Discurso Sujeito Coletivo (DSC) proposto por (Lefèvre e Lefèvre, 2009). O DSC é uma técnica capaz de fazer a organização das opiniões os alunos recolhidas durante o estudo, se utilizando da categorização de trechos do discurso de cada indivíduo observado/entrevistado para a criação de uma ideia que representa a opinião do grupo como um sujeito único. Foram considerados a opinião dos estudantes quanto os fatores de Motivação, Reflexão, Cooperação e Suporte Just-in-Time (Hmelo-Silver, 2004) para análise.

\section{Resultados}

Neste trabalho, para que a opinião dos alunos pudesse ser investigada, uma pergunta relacionada ao acréscimo motivacional proporcionado pelo uso do TutorApp foi realizada. A seguir o DSC das turmas do Estudo de Caso 1, para a pergunta "Como você analisa que a tecnologia utilizada pelo aplicativo, motivou e auxiliou você durante o processo de aprendizagem trabalhado na disciplina ?", são apresentados:

DSC I - "Pude ver que no app recebíamos alguns links e algumas referências de artigos/tutoriais e, mesmo que os links passados estavam tendo que ser digitados para acessá-los, vinham com conteúdos capazes de nos ajudar nos problemas que temos de resolver durante a disciplina. Para quem ainda não sabia foi muito útil. Algumas coisas que foram recebidas nas notificações eu já sabia e por isso esperava ter acesso a tutoriais mais avançados, e nesses momentos, vejo que ajudou parcialmente. Algumas das notificações acabaram aparecendo em momentos onde eu sempre estava ocupado e, infelizmente, quando marcava para ver depois, eu não podia mais avaliar. Achei ótima a proposta do app. Mesmo tendo alguns bugs, a tecnologia acabou ajudando em momentos 
de dificuldade e fez com que eu estudasse em horas que eu normalmente não estudo, fazendo com que eu ampliasse meus conhecimentos."

DSC II - "Foi muito interessante esta forma de obter estímulo para a busca de aprendizagem. Com a chegada de cada notificação, eu me motivava para continuar com os trabalhos da disciplina e buscar mais informações, o que dava produtividade nos meus estudos. Quanto aos materiais que foram indicados pelo aplicativo, foram de grande valia. Algumas vezes o conteúdo da informação poderia ser mais específico, mas no geral ele apresentava dicas dos conteúdos que eu estava tendo dificuldades em encontrar para estudar, sugerindo conteúdo com informações que chegaram no momento certo, como quando me auxiliou informando um conteúdo sobre a área de qualidade, que estava relacionado a minha necessidade. $O$ aplicativo foi de grande ajuda e analiso como uma boa tecnologia. Penso que seja uma boa verificar o tempo entre a distribuição de materiais, visto que em alguns momentos, as sugestões poderiam ter sido sugeridas dias antes. Outro ponto que indico revisar é a forma de como as dicas são apresentadas, pois a aplicação não roda em segundo plano e os links divulgados não podiam ser abertos diretamente pelo aplicativo, sendo necessário copiar caractere a caractere do link no navegador, o que não é tão motivador. A maioria das sugestões do aplicativo foram bem aproveitadas. A tecnologia me ajudou a lembrar de tarefas de modo que quando eu recebia as notificações, lembrava do trabalho a ser feito e já me antecipava ao mesmo. A tecnologia me motivou a realizar o trabalho, buscar o conhecimento sobre minha área de interesse e ir além do material fornecido pelo mesmo."

Abaixo são listadas as análises e discussões referentes ao Estudo de Caso 1:

MOTIVAÇÃO: A motivação é um dos principais fatores para o sucesso no processo de aprendizagem do aluno, bem como um dos maiores objetivos que busca-se alcançar com a prática da PBL. Tendo este fator como base, considera-se que a proposta da ferramenta alcançou o objetivo de motivar o aluno, visto os trechos dos discursos dos alunos a seguir: "(...) Foi muito interessante esta forma de obter estímulo para a busca de aprendizagem (...)"; "(...) Com a chegada de cada notificação, eu me motivava para continuar com os trabalhos da disciplina e buscar mais informações (...)"; e "(...) fez com que eu estudasse em horas que eu normalmente não estudo (...)".

REFLEXÃO: Desenvolver o pensamento reflexivo, que proporcione ao aluno a capacidade de inter-relacionar conteúdos (Souza e Dourado, 2015), não deixando que a informação seja aprendida isoladamente, também é um fator de desafio para a PBL (Hmelo-Silver, 2004). Neste estudo de caso, identificamos que esta prática foi proporcionada e este objetivo alcançado, visto que identificamos trechos do discurso dos alunos como, "(...) fazendo com que eu ampliasse meus conhecimentos (...)", "(...) vinham com conteúdos capazes de nos ajudar nos problemas que temos de resolver durante a disciplina (...)", "(...) materiais que foram indicados pelo aplicativo, foram de grande valia (...)" e "(...) dava produtividade nos meus estudos (...)", que indicam a influência que a aplicação conseguiu alcançar em seu processo de aprendizado.

SUPORTE JUST-IN-TIME: Durante o estudo da PBL apresentado em (Devincenzi et al., 2018), identificamos que o suporte Just-in-Time é extremamente importante para o sucesso da metodologia (Hmelo-Silver, 2004), uma vez que fornecer instruções no momento em que o aluno às necessita é benéfico e extremamente importante para que o processo de aprendizagem seja bem sucedido. Neste ponto, notamos através do experimento, a efetividade da proposta em possibilitar este suporte. Os trechos "(...) a tecnologia acabou ajudando em momentos de dificuldade (...)" e "(..) no geral ele 
apresentava dicas dos conteúdos que eu estava tendo dificuldades em encontrar para estudar, sugerindo conteúdo com informações que chegaram no momento certo, como quando me auxiliou informando um conteúdo sobre a área de qualidade, que estava relacionado a minha necessidade (...)", demonstram evidências de que este suporte ocorreu de forma efetiva, auxiliando os alunos e potencializando seu processo de auto-aprendizado, sempre que eles mostravam a necessidade de um determinado conhecimento ou instrução. Também durante a análise dos resultados apresentados neste estudo de caso, apesar da opinião geral sobre a tecnologia ter sido positiva, "(...) Achei ótima a proposta do app (...)" e "(...) O aplicativo foi de grande ajuda e analiso como uma boa tecnologia (...)", notamos a falta de evidências relacionadas a instigação para a prática de cooperação e também identificamos algumas críticas da ferramenta proposta, em opiniões como:

PERSONALIZAÇÃO DO CONTEÚDO: Notamos através dos trechos de depoimento dos alunos, "(...) Algumas vezes o conteúdo da informação poderia ser mais específico (...)" e "(...) Algumas coisas que foram recebidas nas notificações eu já sabia (...)", que uma evolução da ferramenta para melhor possibilitar a personalização dos materiais enviados, quanto ao seus níveis (básicos e avançados) de aprofundamento nos conteúdos, era necessária.

MOMENTOS DOS DISPAROS: Durante o estudo, com os trechos "(...) verificar o tempo entre a distribuição de materiais (...)", "(...) Algumas das notificações acabaram aparecendo em momentos onde eu sempre estava ocupado (...)" e "(...) em alguns momentos, as sugestões poderiam ter sido sugeridas dias antes (...)", também podemos notar a necessidade de calibrar melhor os disparos dos gatilhos, para que o momento que estes chegam aos alunos, sejam melhor alcançados.

BUGS DA FERRAMENTA: Por fim, com os alertas detectados nos trechos "(...) links divulgados não podiam ser abertos diretamente (...)" e "(...) links passados estavam tendo que ser digitados para acessá-los (...)", podemos identificar bugs que a ferramenta apresentava e que deveriam ser corrigidos.

Após o Estudo de Caso 1, as análises feitas foram utilizadas para evoluir a ferramenta Anônimo. Um novo experimento (Estudo de Caso 2) foi proposto e após sua conclusão, novamente aplicamos a pergunta "Como você analisa que a tecnologia utilizada pelo aplicativo, motivou e auxiliou você durante o processo de aprendizagem trabalhado na disciplina ?", a qual resultou o seguinte:

DSC III - "A ideia do TutorApp é muito boa. Os materiais foram enviados com sucesso e quando precisávamos, mostrando que a tecnologia utilizada foi muito eficaz. É uma ferramenta com potencial, me deu um bom guia e conteúdo complementar para começar a estudar, e mesmo que não houvesse possibilidades de leitura no momento que o material foi recebido, a pendência da leitura continua sendo uma forma de incentivo. Quanto a usabilidade do TutorApp, não é muito intuitivo. Faltou um menu mais detalhado de ajuda do que foi disponibilizado, tive dificuldades para identificar qual campo eu deveria preencher, principalmente com explicações do Quadro Branco. Outra dificuldade encontrada foi a falta de notificação dos gatilhos, apenas se eu entrasse no aplicativo aparecia algo e muitas vezes não conseguia acessar os links. $O$ aplicativo me auxiliou em alguns pontos superficialmente, como ponto de partida para uma pesquisa mais aprofundada, os PDF's disponibilizados também são ótimos, porém foi necessário pesquisar mais pelo conteúdo, visto que o fornecido não sanou todas as minhas dúvidas. As notificações não foram muito invasivas e acabam funcionando como alarme para lembrar determinado assunto, o que auxiliou no processo de aprendizagem e foi 
interessante. Contudo necessita de mais presença do professor, tanto para auxiliar com o uso do app, quanto para solucionar dúvidas. Os gatilhos foram motivacionais, incentivando o estudo, na grande maioria das vezes com informações convenientes, sempre lembrando de me manter em atividade, o que gera uma continuidade de pesquisa."

Abaixo são listadas as análises e discussões referentes ao Estudo de Caso 2:

MOTIVAÇÃO: Observamos que a PBL é uma metodologia pautada em esforços para elevar este grau de motivação dos alunos para garantir o sucesso em seu processo de aprendizado. Assim, consideramos que este estudo alcançou o objetivo de motivar o aluno, visto os trechos dos discursos dos alunos a seguir: "(...) As notificações não foram muito invasivas e acabam funcionando como alarme para lembrar determinado assunto (...)", "(...) gatilhos foram motivacionais, incentivando o estudo (...)", "(...) grande maioria das vezes com informações convenientes (...)" e "(...) sempre lembrando de me manter em atividade, o que gera uma continuidade de pesquisa (...)". Ainda relacionado aos ganhos de motivação alcançados pelo uso da tecnologia, destacamos o trecho do discurso dos alunos "(...) mesmo que não houvesse possibilidades de leitura no momento que o material foi recebido, a pendência da leitura continua sendo uma forma de incentivo (...)" para evidenciar o ganho que obtivemos em agregar ao processo de aprendizagem do aluno a potencialidade motivacional que as tecnologias utilizadas proporcionaram.

REFLEXÃO: Destacamos importância da promoção do pensamento reflexivo no aluno, capacitando-o a inter-relacionar conteúdos (Hmelo-Silver, 2004). Analisando a execução deste experimento, identificamos que esta promoção da reflexão, constituiu-se em um dos objetivos alcançados por nossa pesquisa, onde destacamos aqui, para contribuir com essa afirmação, trechos do discurso dos alunos como "(...) auxiliou em alguns pontos superficialmente, como ponto de partida para uma pesquisa mais aprofundada (...)" e "(...) me deu um bom guia e conteúdo complementar para começar a estudar (...)", que demonstram a forma como as instigações e os conteúdos disponibilizados via disparos de objetos de aprendizado, estavam adequados a desafiar eles a pensar e refletir. Vale destacar também aqui, o trecho "(...) porém foi necessário pesquisar mais pelo conteúdo, visto que $o$ fornecido não sanou todas as minhas dúvidas (...)", que demonstra o quanto os materiais foram precisos em não apresentar, em determinados momentos, conteúdos que contemplam toda a informação necessária ao aluno, mas sim o nível certo desta informação para que este aluno seguisse sendo desafiado a investigar e refletir sobre as soluções do problema.

SUPORTE JUST-IN-TIME: Também a necessidade de fornecer instruções no momento em que 0 aluno às necessita (Just-in-Time), para que o processo PBL tenha sucesso em sua execução (Hmelo-Silver, 2004). Para este estudo de caso, através do uso da estrutura "Quadro Branco" implementada na ferramenta, notamos ter ampliado o alcance da necessidade momentânea do aluno, visto que, sempre que o aluno cadastrou na ferramenta seus "Fatos", suas "Ideias" e seus "Pontos de Aprendizagem", conseguimos identificar um ponto de necessidade no momento, e assim disparar objetos de aprendizado com suporte a esta necessidade. O trecho "(...) materiais foram enviados com sucesso $e$ quando precisávamos (...)" auxilia a comprovar nossa percepção quanto ao alcance do suporte Just-in-Time.

COOPERAÇÃO: No Estudo de Caso 1, não foram percebidas evidências de esforços na ferramenta implementada para avaliar a tecnologia proposta. Neste segundo estudo de caso, com a criação da estrutura "Quadro Branco", buscou-se criar um ambiente 
que fosse favorável a cooperação entre os alunos, como sendo de extrema importância ao sucesso do processo PBL (Hmelo-Silver, 2004). Acredita-se na potencialidade da estrutura "Quadro Branco" em promover cooperação entre os alunos, visto que ela disponibilizou a possibilidade de cadastro, recebimento e consulta de materiais a todos alunos pertencente a um grupo de trabalho, e em complemento, a possibilidade de consulta e acompanhamento destas informações pelos outros grupos da disciplina.

Ao analisar os depoimentos dos alunos, notamos os seguintes trechos "(...) tive dificuldades para identificar qual campo eu deveria preencher, principalmente com explicações do Quadro Branco (...)" e "(...) necessita de mais presença do professor, tanto para auxiliar com o uso do app, quanto para solucionar dúvidas (...)", que surgem como possíveis motivos para a falta de evidências quanto a promoção de cooperação no experimento. Concluindo a discussão dos resultados apresentados neste estudo de caso, notamos, através dos seguintes trechos "(...) A ideia do TutorApp é muito boa (...)" e "(...) a tecnologia utilizada foi muito eficaz (...)" que a opinião geral sobre a tecnologia foi positiva. Entretanto algumas deficiências técnicas da ferramenta foram relatadas em trechos como, "(...) a usabilidade do TutorApp, não é muito intuitivo (...)", "(..) Faltou um menu mais detalhado de ajuda (...)", "(...) falta de notificação dos gatilhos, apenas se eu entrasse no aplicativo aparecia algo (...)" e "(...) muitas vezes não conseguia acessar os links (...)". Ressaltamos aqui, que estas deficiências devem ser investigadas e corrigidas, antes da utilização da ferramenta em projetos futuros.

\section{Considerações Finais}

A técnica do DSC mostrou-se viável durante o experimento, pois possibilitou organizar as opiniões dos alunos coletadas durante esse estudo. Observar as concepções do grupo como um sujeito único permitiu que pudemos responder os questionamentos a respeito de como as TPs i) auxiliam momentos de reflexão no momento adequado; ii) como provêem o suporte just-in-time; iii) como auxiliam no processo de promoção da cooperação entre os alunos; e por fim, iv) como podem elevar o grau de motivação do aluno durante 0 seu processo de aprendizagem. As opiniões, em síntese, revelam que os alunos se sentiram motivados e desafiados a partir do recebimento dos gatilhos. Fato que se justifica devido ao envio destes terem proporcionado questionamentos e a ampliação dos processos reflexivos sobre o tema no momento adequado a dinâmica da aprendizagem. Podemos notar o grau de satisfação dos alunos, quanto ao suporte dado pela ferramenta às necessidades de conteúdos que estes alunos tinham naquele momento (caráter ubíquo da ferramenta). Estes trechos também apontaram que a cada gatilho disparado, corroboraram com a conclusão de que TP e Ubíquas, quando utilizadas juntas, podem contribuir para elevar a motivação do aluno durante a prática PBL. Com a análise dos discursos também pudemos elencar pontos que necessitavam ajustes na ferramenta. Além do aumento de motivação dos alunos em relação a atividade/ferrament proposta, associada ao processo de detectar dúvidas e incertezas do aluno e disparar objetos de aprendizado com suporte a esta necessidade.

\section{Agradecimentos}

Universidade Federal do Rio Grande, Instituto Federal do Rio Grande do Sul. O presente trabalho foi realizado, em parte, com apoio da Coordenação de Aperfeiçoamento de Pessoal de Nível Superior - Brasil (CAPES) - Código de Financiamento 001. 


\section{Referências Bibliográficas}

BARROWS, H. S. A taxonomy of problem-based learning methods, Medical education. vol. 20, pp. 481-486, 1986.

DEVINCENZI, S. ; TOLÊDO, F. P. ; KWECKO, V. ; MOTA, F. P. ; BOTELHO, S. S. C. Ubiquitous Environments for Problem-Based Learning: A Bibliographic Review. In IEEE Frontiers in Education Conference (FIE) (pp. 1-6). IEEE. 2018

DEVINCENZI, S. ; TOLÊDO, F. P. ; KWECKO, V. ; CASARIN, J. ; BOTELHO, S. S. C. $O$ uso de tecnologias persuasivas para potencializar o processo de aprendizagem baseado em problemas. Espacios, vol 38, №60, pp 13-23, 2017.

DIJKSTRA, A. "Personalization/computer-tailoring in persuasive technology: Tailoring ingredients target psychological processes," Proceedings of the International Workshop on Personalization in Persuasive Technology (PPT'16), Salzburg, Austria, 2016.

FILLIPOU, J. ; CHEONG, C. ; CHEONG, F. Designing persuasive systems to influence learning: Modelling the impact of study habits on academic performance, PACIS 2015, 2015.

FOGG, B. J. A behavior model for persuasive design, in Proceedings of the 4th international Conference on Persuasive Technology, p. 40, ACM, 2009.

SOUZA, S. C. De; DOURADO L. Aprendizagem baseada em problemas (abp): um método de aprendizagem inovador para o ensino educativo, Holos, vol. 5, pp. 182-200, 2015.

HMELO-SILVER C. E. Problem-based learning: What and how do students learn?, Educational psychology review, vol. 16, no. 3, pp. 235-266, 2004.

HWANG G.-J. ; YANG, T.-C. ; TSAI C.-C. ; YANG S. J. A context aware ubiquitous learning environment for conducting complex science experiments, Computers \& Education, vol. 53, no. 2, pp. 402-413, 2009.

$\mathrm{KU}$, D. T. ; CHANG C.-S. A framework of pbl strategy integrated in Ims and a ubiquitous learning environment, in Networked Computing and Advanced Information Management (NCM), 2010 Sixth International Conference on, pp. 722-726, IEEE, 2010.

LEFÈVRE, F. ; LEFÈVRE A. M. C. Pesquisa de representação social: um enfoque qualiquantitativo: metodologia do discurso do sujeito coletivo, in Pesquisa de representação social: Um enfoque qualiquantitativo: metodologia do discurso do sujeito coletivo, 2010.

SANTAELLA L. A aprendizagem ubíqua na educação aberta, Revista Tempos e Espaços em Educação, pp. 15-22, 2014. 